\title{
Escape from Vela X
}

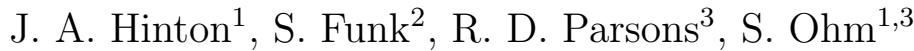

\begin{abstract}
Whilst the Vela pulsar and its associated nebula are often considered as the archetype of a system powered by a $\sim 10^{4}$ year old isolated neutron star, many features of the spectral energy distribution of this pulsar wind nebula are both puzzling and unusual. Here we develop a model that for the first time relates the main structures in the system, the extended radio nebula (ERN) and the Xray cocoon through continuous injection of particles with a fixed spectral shape. We argue that diffusive escape of particles from the ERN can explain the steep Fermi-LAT spectrum. In this scenario Vela $\mathrm{X}$ should produce a distinct feature in the locally-measured cosmic ray electron spectrum at very high energies. This prediction can be tested in the future using the Cherenkov Telescope Array (CTA). If particles are indeed released early in the evolution of PWNe and can avoid severe adiabatic losses, PWN provide a natural explanation for the rising positron fraction in the local CR spectrum.
\end{abstract}

Subject headings: Gamma rays: general, Gamma rays: ISM, (ISM:) cosmic rays, (Stars:) pulsars: individual: PSR B0833-45

\section{Introduction}

The proximity (distance: 290 pc, measured by parallax Caraveo et al. 2001; Dodson et al. 2003) of the Vela pulsar (PSR B0833-45) has allowed it to be studied in great detail across the whole electromagnetic spectrum. PSR B0833-45 (spin-down power $\dot{E}=7 \times$ $10^{36} \mathrm{erg} / \mathrm{s}$, characteristic age $\tau_{c}=11,000$ years (Manchester et al. 2005)) is embedded in a diffuse radio nebula (Large et al. 1968). Higher angular-resolution radio (Dwarakanath 1991; Duncan et al. 1996) and X-ray (Kahn et al. 1985; Aschenbach et al. 1995) observations

\footnotetext{
${ }^{1}$ Department of Physics and Astronomy, University of Leicester, Leicester LE1 7RH, UK

${ }^{2}$ Kavli Institute for Particle Astrophysics and Cosmology, SLAC, 2575 Sand Hill Road, Menlo Park, CA-94025, USA, funk@slac.stanford.edu

${ }^{3}$ School of Physics \& Astronomy, University of Leeds, Leeds LS2 9JT, UK
} 
of the region established the presence of (1) a large circular shell (about $8^{\circ}$ in diameter) the Vela SNR - and (2) an extended radio nebula (ERN) of size $2^{\circ} \times 3^{\circ}$ in the centre dubbed Vela X (see Figure 1 left). Based on the high degree of polarisation and the flat radio spectral index Weiler \& Panagia (1980) suggested that Vela X is a pulsar wind nebula (PWN) powered by PSR B0833-45. Such PWNe act as calorimeter, containing the timeintegrated particle outflow and magnetic field from the pulsar. The radio emission is far from uniform, exhibiting a network of filaments throughout the nebula (Frail et al. 1997) . The brightest of these non-thermal filamentary structures in Vela X emerges to the south of PSR B0833-45 and has a size of $45^{\prime} \times 12^{\prime}$ (Figure 1 (right). The X-ray counterpart to this bright radio filament was detected using ROSAT (Markwardt \& Ögelman 1995) (see Figure 1 right) and dubbed "the cocoon". It is thought to be the result of the interaction of the PWN with the SNR reverse shock (Blondin et al. 2001).

X-ray observations performed by Mangano et al. (2005) found that within $1^{\prime}$ of the Vela pulsar the 3-10 keV spectrum softens with increasing distance, a signature of electrons cooling via synchrotron emission. BeppoSax data demonstrated that the nebula emission in the inner $12^{\prime}$ in radius extends up to $\sim 200 \mathrm{keV}$ (Mangano et al. 2005) with most of the emission coming from within $4^{\prime}$ of the pulsar, suggesting particle acceleration close to the pulsar (black circle in Figure 1).

H.E.S.S. detected $\mathrm{TeV} \gamma$-ray emission from Vela X exhibiting an atypical spectrum with energy flux peaking at $\sim 10 \mathrm{TeV}$ (Aharonian et al. 2006). The $\mathrm{TeV}$ emission is dominated by an elliptical region of $58^{\prime} \times 43^{\prime}$, extending significantly beyond the X-ray cocoon, yet smaller than the ERN (see Figure 1 right). The latest H.E.S.S. results (Dubois et al. 2009), show significant, but low-surface brightness TeV emission beyond this region. Perhaps surprisingly, the $\gamma$-ray spectrum in the outer parts is identical to that of the central region. The H.E.S.S. detection of emission from the cocoon provided the first robust estimate of the magnetic field strength in this region: $\sim 4 \mu \mathrm{G}$. No excess $\mathrm{TeV}$ emission was seen from the hard X-ray emitting region close to the pulsar, implying a magnetic field of $B>50 \mu \mathrm{G}$ in the immediate vicinity of the pulsar, consistent with the cooling signature detected with BeppoSax.

Finally, Fermi-LAT observations in the Vela pulsar off-pulsa data established the presence of an extended $\gamma$-ray emitting structure at energies above $800 \mathrm{MeV}$ spatially coincident with the ERN (see Figure 1 left) with a spectral index of $\sim 2.4$ (Abdo et al. 2010). The two-peaked nature of the $\gamma$-ray spectral energy distribution (SED) led the authors to conclude (following an earlier suggestion by de Jager et al. 2008) that there are two distinct populations of electrons, one responsible for the radio and $\mathrm{GeV} \gamma$-ray emission and the other for the X-ray and $\mathrm{TeV}$ emission. The magnetic field derived through these observations is $\sim 4 \mu \mathrm{G}$. 


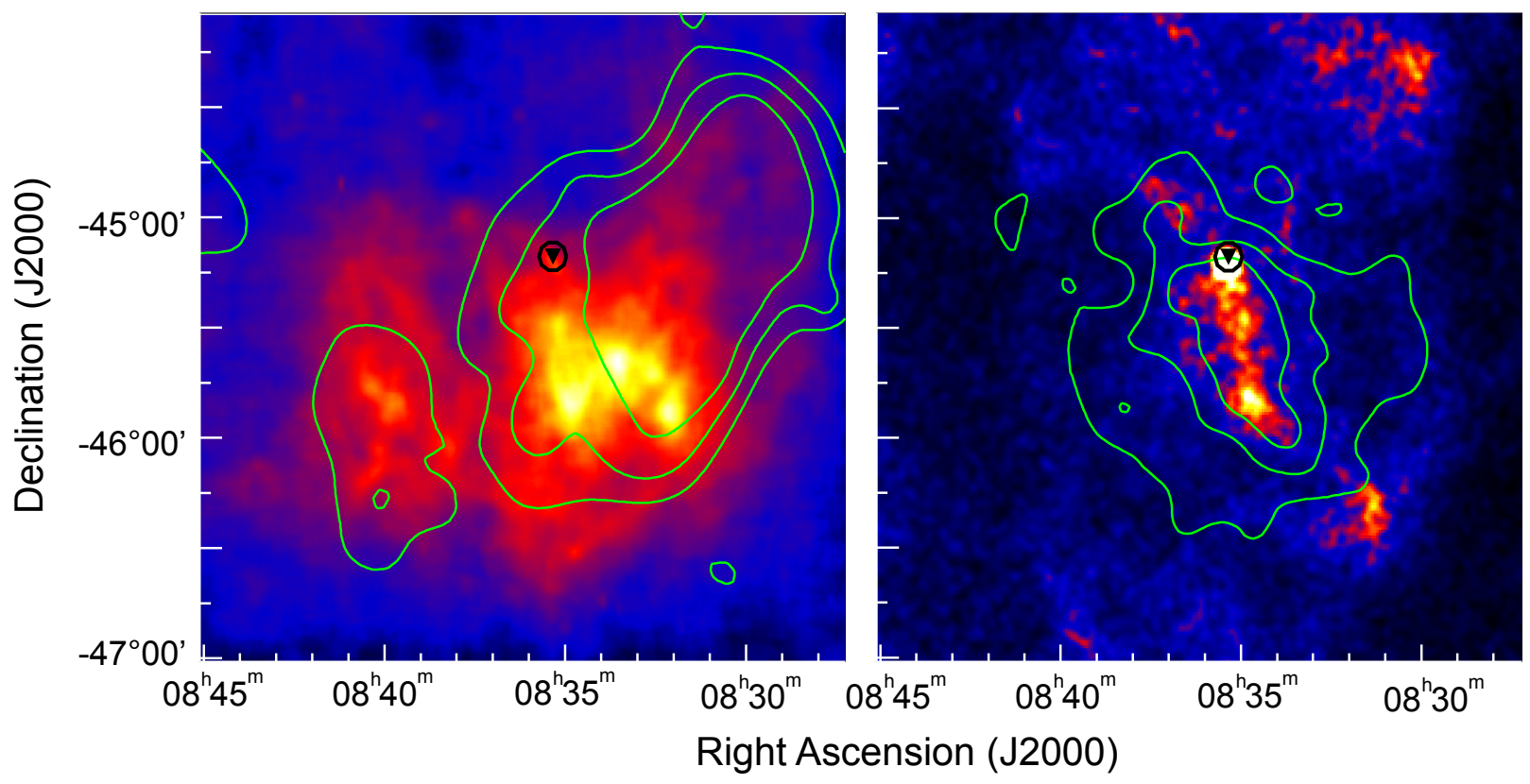

Fig. 1.- Left: Radio image of a $3^{\circ} \times 3^{\circ}$ region around Vela (black triangle), from $8 \mathrm{GHz}$ VLA data (Frail et al. 1997). Overlaid are Fermi-LAT 4,5 and $6 \sigma$ significance contours (green) above $800 \mathrm{MeV}$ (Abdo et al. 2010). Right: ROSAT (0.9-2.4 keV) count map (Markwardt \& Ögelman 1997), smoothed with a Gaussian kernel of 2.25', saturated at $10 \%$ of the pulsar peak intensity. Overlaid are H.E.S.S. smoothed excess contours $(25,50,75 \%$ of the peak emission) (Aharonian et al. 2006). The black circle indicates the 4' region where most of the hard X-ray emission originates (Mangano et al. 2005).

Despite the wealth of experimental data and several theoretical studies, many questions remain about the relationship between the emission seen on different scales in Vela X. In particular, there are several important observational facts for which no satisfactory explanation has yet been put forward. Firstly, the steep GeV spectrum, indicating an unexplained absence of $>100 \mathrm{GeV}$ energy particles from the ERN. Secondly, the relative dimness of the $\mathrm{TeV}$ Nebula. Comparing the spin-down luminosity of the Vela pulsar to the $\mathrm{TeV}$ luminosity above $500 \mathrm{GeV}\left(10^{33} \mathrm{erg} / \mathrm{s}\right)$ yields an efficiency in the cocoon of $0.01 \%$, significantly lower than typical TeV PWNe (Aharonian et al. 2006). In addition, the lack of spectral variations across Vela $\mathrm{X}$ above $1 \mathrm{TeV}$ is not readily explained in any model in which the $\mathrm{TeV}$ size is limited by cooling.

Here we show that a scenario in which the high energy particles have diffused out of the ERN, and where the cocoon is a relatively recent feature of the PWN, may answer these questions. This scenario has implications for the locally measured electron spectrum at high 
energies. It has been pointed out (Shen 1970) that only very local sources can significantly contribute to the flux of cosmic ray electrons above $1 \mathrm{TeV}$, with Vela (and Geminga) long seen as likely candidates (see e.g. Nishimura et al. 1980; Atoyan et al. 1995; Kobayashi et al. 2004). The recent measurements of a rise in the positron fraction above $\sim 10 \mathrm{GeV}$ (Adriani et al. 2009; Ackermann et al. 2011) indicate that a relatively local source of positrons must be present. Pulsars and their nebulae as plausible candidates (see e.g. Chi et al. 1996; Büsching et al. 2008; Profumo 2008; Yüksel et al. 2009), given that we see high-energy electrons/positrons radiation at $\mathrm{TeV}$ energies. However, the details of the escape of particles from the PWN region have been left open. Here we demonstrate for the first time how the signature of escape may be directly visible in the SED of Vela X.

\section{Model}

We explore the possibility that particle propagation effects dominate the spectral and morphological features of Vela X. We attempt to reconcile the properties of the $\mathrm{TeV}$ and $\mathrm{GeV}$ nebula by considering an electron injection from the pulsar wind termination shock which represents a fixed fraction of the spin-down power of the pulsar and has a fixed spectral shape. The two $\gamma$-ray-emitting zones of Vela $\mathrm{X}$ are discussed in turn.

The TeV Nebula: Whilst the TeV emission of Vela $\mathrm{X}$ is now known to extend over much of the ERN region, the bulk of the TeV-emitting particles appear to be confined to a small fraction of its the volume. This emission is peaked in the cocoon, but a consistent spectral shape is measured throughout the ERN. This suggests that the electron population of the cocoon is not cooling-limited in extent and that the probability of escape from the system is very small and/or energy-independent. These properties appear consistent with the paradigm of (at least young) PWNe as internally advection-dominated systems from which particles cannot easily escape (Aharonian et al. 1997). The energetic particle content should then be consistent with the power supplied by the pulsar during the period of injection into the cocoon. As the energetic particle content of Vela X is well-determined by $\mathrm{TeV}$ and X-ray observations, the age of the $\mathrm{TeV}$ cocoon can be expressed as: $t_{\mathrm{coc}}=E_{\mathrm{coc}} /\left(\dot{E}_{\epsilon}\right) \approx 70 \mathrm{yrs} / \epsilon$, with $E_{\text {coc }} \sim 1.5 \times 10^{46} \mathrm{erg}$ (Abdo et al. 2010). Whilst there are considerable uncertainties on the efficiency factor $\epsilon$ of converting spin-down power into relativistic electrons for any plausible value of $\epsilon$, the cocoon is much younger than the system as a whole. This is consistent with an interpretation of the cocoon as a feature arising from the interaction with the SNR reverse shock (Blondin et al. 2001). The maximum energy of the cocoon particles is constrained by the H.E.S.S. measurements to $\sim 70 \mathrm{TeV}$ (Aharonian et al. 2006). This 
maximum energy has been interpreted as a consequence of cooling of higher energy particles in the cocoon (de Jager 2008; Abdo et al. 2010). However, the synchrotron lifetime for $70 \mathrm{TeV}$ electrons is $t_{\mathrm{sync}}=10^{4}(B / 4 \mu \mathrm{G})^{-2}$ years, consistent with the age of the Vela pulsar but not with the lifetime of the cocoon. We hypothesize that the maximum energy of cocoon particles is determined by cooling in the high- $B$-field $(\sim 100 \mu \mathrm{G})$ region in the immediate $(\sim 0.5 \mathrm{pc})$ vicinity of the pulsar before injection into the low- $B$-field cocoon region. The $O(10 \mathrm{yr})$ residence time required for a cooling break to appear at $70 \mathrm{TeV}$ for particles emerging from the high B-field region is consistent with expectations for the post-shock flow (see e.g. Mangano et al. 2005).

The Extended Radio Nebula It seems likely that the ERN contains particles injected over the lifetime of the system, up to the evolution stage at which the cocoon appeared. In contrast to the cocoon, evolutionary effects in the spin-down power of the pulsar become critical in determining the available energy. The total energy injected into the ERN depends critically on the assumed birth-period of the pulsar $\left(P_{0}\right)$. The energy in relativistic particles is $\sim 2 \times 10^{49} \epsilon\left(P_{0} / 30 \mathrm{~ms}\right)^{-2}$. The electron content of the ERN has been estimated to be $5 \times 10^{48}$ erg (Abdo et al. 2010) using the similarity in radio and $\mathrm{GeV} \gamma$-rays. Birth-periods as high as $P_{0}=40 \mathrm{~ms}$ have been discussed (van der Swaluw \& Wu 2001) to account for the ratio of PWN radius to SNR radius but require $\epsilon$ close to 1 . No explanation has been put forward for the absence of $>100 \mathrm{GeV}$ particles in the ERN required by the steep Fermi-LAT spectrum. Given the current-day magnetic field of $\sim 4 \mu \mathrm{G}$, the cooling times for particles emitting in the GeV-range are very large and synchrotron cooling seems implausible. While strong evolution of the magnetic field in PWNe is possible (see e.g. Gelfand et al. 2009), an implausibly high effective time-averaged B-field of $>100 \mu \mathrm{G}$ within the whole ERN is required to explain the Fermi-LAT GeV spectrum with a cooling break. Since there is no obvious theoretical reason why the maximum accelerated energy from the pulsar should be time-dependent, the remaining explanation is that the bulk of the $>100 \mathrm{GeV}$ particles have escaped from the ERN into the interstellar medium (ISM). Whilst confinement of particles in PWNe is thought to be effective in their early evolution it is likely that the interaction with the SNR reverse shock which seems to have appeared in Vela X a few thousand years ago (Blondin et al. 2001; Gelfand et al. 2009) brings an end to the effective confinement of electrons. Such a reverse shock interaction is expected to disrupt the PWN sufficiently, through e.g. the growth of Rayleigh-Taylor instabilities, that diffusion of particles out of the PWN becomes possible. This explanation for the absence of $>100 \mathrm{GeV}$ particles in the ERN requires a diffusion coefficient of $D=R_{\mathrm{pc}}^{2} / 2 t_{\mathrm{kyr}} \sim \times 10^{26} \mathrm{~cm}^{2} \mathrm{~s}^{-1}(R$ in parsec, $t$ in units of kyears). For typical parameters in Vela $\mathrm{X}$ this is of order 1000 times slower than that inferred for cosmic rays in the ISM, but much faster than Bohm diffusion for the magnetic 
field in the ERN (Aharonian 2004).

Calculation For the calculation of the present-day SED of Vela X and the energy and timedependent flux of escaping particles we employ a Monte-Carlo approach where propagation and energy losses of individual particles are considered. Following earlier studies we neglect adiabatic losses due to the likely return of most of the lost energy at the time of reverse shock crushing of the PWN (see e.g. de Jager et al. 2008). Synchrotron and Klein-Nishina (KN) inverse Compton (IC) losses are calculated at each time step. For the calculation of IC cooling/radiation we adopt the soft-photon fields used by de Jager et al. (2008) and Abdo et al. (2010), with black-body spectra of temperatures $25 \mathrm{~K}$ and $6500 \mathrm{~K}$ and energy densities of $0.4 \mathrm{eV} \mathrm{cm}^{-3}$. In the first stage of evolution of the system confinement is assumed to be $100 \%$ effective and a one-zone approach (following the treatment in Hinton \& Aharonian 2007 ) is employed. A canonical braking index of 3 (de Jager 2007) is assumed over the evolution of the pulsar, rather than the value measured for the recent past (determined to be $1.6 \pm 0.1$ by Dodson et al. 2003). The choice of this value has very little impact on the final model curves. For $P_{0}=30 \mathrm{~ms}$, the implied age of the system is $t_{\text {sys }}=10$ kyears. At $t_{\mathrm{rs}}=\sim 70 \% t_{\mathrm{sys}}$ (the age when the reverse shock is thought to start interaction with the ERN, see e.g. Blondin et al. 2001), diffusive transport is switched on, with particles initially uniformly distributed in a spherical PWN, with continued injection until $t_{\mathrm{sys}}-t_{\mathrm{coc}}\left(t_{\mathrm{coc}}=230\right.$ years, $\epsilon=0.3$ ), from which time particles injected at the termination shock are assumed to be confined within the cocoon. This is consistent with simulations (e.g. van der Swaluw et al. 2004) in that when the reverse shock has collided with the PWN, the pulsar no longer injects particles into the "relic PWN" but forms a new PWN. Diffusion is assumed to be a fixed factor $\delta$ faster than Bohm diffusion, with the magnetic field in the nebula falling continuously with $t^{-0.5}$ at late times. The free parameters of the model are the pulsar birth period $P_{0}$, the efficiency $\epsilon$ of conversion of spin-down power to particles in the MeV-100 TeV domain, the factor $\delta$ and the current-day ERN magnetic field. For the TeV nebula the magnetic field strength and cocoon age form the additional free parameters. To model propagation and energy losses of the electrons released into the ISM we adopt an analytical solution of the diffusion-loss equation (Atoyan et al. 1995), assuming synchrotron and IC radiation as the dominant loss mechanisms. Synchrotron losses assume a local ISM magnetic field strength of $5 \mu \mathrm{G}$. The IC losses were calculated using the full $\mathrm{KN}$ description, assuming 4 radiation fields (CMB, IR, G-K stars and O-B stars) with a total radiation density of $1 \mathrm{eV} \mathrm{cm}{ }^{-3}$. 


\section{Discussion}

Figure 2 shows measured and calculated SEDs for the ERN (red) and the TeV cocoon (blue). There is a striking inconsistency between the WMAP measurement shown in (Abdo et al. 2010) and earlier radio measurements (Alvarez et al. 2001; Hales et al. 2004). We attempt to approximately match the spectral shapes, rather than the $\sim 10^{-4} \mathrm{eV}(50 \mathrm{GHz})$ normalisation, given the apparent factor $\sim 3$ disagreement. Data from Planck should resolve this issue in the near future. The main impact on our model is a change in the current-day magnetic field.

As previously discussed, cooling effects are not important inside the cocoon (blue curves). The spectrum in the cocoon is therefore unmodified from the injection spectrum. The assumption is that the cooling of the particles inside the cocoon has happened close to the pulsar, consistent with the low magnetic field $(\sim 4 \mu \mathrm{G})$ in the cocoon and the much higher field $(\sim 100 \mu \mathrm{G})$ close to the pulsar. Thus, this curve is similar to the time-independent one-zone IC model shown in Aharonian et al. (2006).

The expected ERN flux (red) in the absence of particle escape for constant efficiency $\epsilon$ is shown as dashed lines in Fig. 2, clearly demonstrating how dramatically different the observed spectrum in the Fermi-LAT energy range is. In this case the peak energy and fluxes are determined by particle cooling. The solid lines show the energy-escape based models under the approximation of spherical symmetry and a $7 \mathrm{pc}$ radius. Different curves illustrate the effects of different diffusion coefficients. The apparently best-match to the data yields a factor of $\delta=2000$ faster than Bohm diffusion (with the same $D \propto E^{\delta}, \delta=1$ energy dependence). Lower exponents $\delta=0.6$ produce acceptable fits with modifications of the model parameters within a reasonable range, whilst significantly lower values seem to be excluded in this scenario. We emphasize that these are illustrative models rather than an attempt at fully describing the system. The details depend critically on the time- and spatial evolution. The apparent small-scale differences between the morphology in the radio and $\mathrm{GeV} \gamma$-ray bands (Figure 1 left) indicate highly non-uniform magnetic fields in Vela X. The true situation of escape of particles out of the ERN is likely highly complex.

Figure 3 (left) shows the expected local electron spectrum from our model, assuming nominal diffusion parameters (black line, see caption for details). Several key parameters affect the local CR electron signature of a source: the distance, the electron injection time and the diffusion coefficient. We investigate the uncertainty on these parameteres by using a simple burst-like injection into the ISM and a spectrum adapted to match the signature of the full calculation $\left(E^{-1.8} \exp (E / 5 \mathrm{TeV})\right.$ with an energy input of $\left.6.8 \times 10^{48} \mathrm{erg}\right)$. Figure 3 (left) shows in red the effect of varying the time at which this burst of electrons is released into the ISM. It can be seen that for an injection time $t_{\text {inj }}=3 \mathrm{ky}$, burst-like injection produces 
similar results to the time-dependent injection model. Figure 3 (right) shows the effects of varying the distance to Vela X within the allowed errors (blue curves) (Dodson et al. 2003) and varying the diffusion coefficient (green curve). If source and diffusion parameters are changed from the nominal model it becomes difficult to keep the spectrum consistent with the H.E.S.S. measurements. Due to the very recent injection of a large number of high energy electrons, Vela X will produce a distinct feature in the local electron spectrum. Such a signature should be easily observable by instruments like CTA (Hermann et al. 2007), expected to achieve a factor $>10$ increase compared to H.E.S.S. in collection area for wellcontained/high-telescope-multiplicity events. Combined with an effective FoV solid angle increase of a factor $>4$ and $\sim 5$ times longer observation time (the published H.E.S.S. data represents $\sim 150 \mathrm{~h}$ ), the expected increase in electron statistics (with comparable systematics and background levels) is a factor $\sim 300$, extending the spectrum from $\sim 4 \mathrm{TeV}$ up to $\sim 70 \mathrm{TeV}$ for an $\mathrm{E}^{-3}$ spectrum.

\section{Conclusions}

Due to its proximity and well-known distance, Vela X presents a unique opportunity to constrain models using excellent spectral and morphological measurements and the locallymeasured electron/positron flux. The model presented here, of energy-dependent escape from Vela X, produces a clear signature in the CR electron spectrum, which could be measured in detail with CTA. If this scenario proves correct and Vela X proves to be typical of PWN of this age, the release of $>\mathrm{TeV}$ particles from PWN relatively early in their evolution may help to explain the rise in the CR positron fraction seen at high energies and at the same time help understand the absence of the significant population of Fermi relic-PWN that had been predicted before launch (see e.g. de Jager 2008).

We thank A. Hales for providing the $8.4 \mathrm{GHz}$ reprocessed image of (originally provided by D. Bock). We thank Felix Aharonian and an anonymous referee for helpful comments. SO acknowledges support by a Humboldt foundation Feodor-Lynen fellowship.

\section{REFERENCES}

Abdo, A. A., Ackermann, M., Ajello, et al., 2010, ApJ, 713, 146

Abdo, A. A., Ackermann, M., Ajello, M., et al., 2009, PRL, 102, 181101

Ackermann, M., Ajello, M., Allafort, A., et al., 2011, ArXiv/1109.0521 
Adriani, O., Barbarino, G. C., Bazilevskaya, G. A., et al., 2009, Nature, 458, 607

Aharonian, F. A., Atoyan, A. M., \& Kifune, T., 1997, MNRAS, 291, 162

Aharonian, F. A. 2004, Very High Energy Cosmic Gamma Radiation (World Scientific Pub Co Inc)

Aharonian, F., Akhperjanian, A. G., Barres de Almeida, U., et al., 2008, PRL, 101, 261104

Aharonian, F., Akhperjanian, A. G., Bazer-Bachi, A. R., et al., 2006, A\&A, 448, L43

Alvarez, H., Aparici, J., May, J., \& Reich, P. 2001, A\&A, 372, 636

Aschenbach, B., Egger, R., \& Trumper, J. 1995, Nature, 373, 587

Atoyan, A. M., Aharonian, F. A., \& Völk, H. J. 1995, Phys. Rev. D, 52, 3265

Blondin, J. M., Chevalier, R. A., \& Frierson, D. M. 2001, ApJ, 563, 806

Büsching, I., de Jager, O. C., Potgieter, M. S., \& Venter, C. 2008, ApJ, 678, L39

Caraveo, P. A., De Luca, A., Mignani, R. P., \& Bignami, G. F. 2001, ApJ, 561, 930

Chi, X., Cheng, K. S., \& Young, E. C. M. 1996, ApJ, 459, L83+

de Jager, O. C. 2007, ApJ, 658, 1177

-. 2008, ApJ, 678, L113

de Jager, O. C., Slane, P. O., \& LaMassa, S. 2008, ApJ, 689, L125

Dodson, R., Legge, D., Reynolds, J. E., \& McCulloch, P. M. 2003, The Astrophysical Journal, 596, 1137

Dubner, G. M., Green, A. J., Goss, W. M., Bock, D. C.-J., \& Giacani, E. 1998, AJ, 116, 813

Duncan, A. R., Stewart, R. T., Haynes, R. F., \& Jones, K. L. 1996, MNRAS, 280, 252

Dwarakanath, K. S. 1991, A\&A, 12, 199

Egberts, K. et al. (H.E.S.S. Collaboration), 2011, NIM A, 630, 36

Frail, D. A., Bietenholz, M. F., Markwardt, C. B., \& Oegelman, H. 1997, ApJ, 475, 224

Gelfand, J. D., Slane, P. O., \& Zhang, W. 2009, ApJ, 703, 2051

Hales, A. S., Casassus, S., Alvarez, H., et al., 2004, ApJ, 613, 977 
Hermann, G., Hofmann, W., Schweizer, T., Teshima, M., \& CTA consortium, f. t. 2007, ArXiv e-prints, 709

Hinton, J. A. \& Aharonian, F. A. 2007, ApJ, 657, 302

Dubois, F., B. Glück, Jager, O. C. D., et al., 2009, Proceedings of the 31st ICRC in Lodz, 45

Kahn, S. M., Gorenstein, P., Harnden, F. R., \& Seward, F. D. 1985, ApJ, 299, 821

Kobayashi, T., Komori, Y., Yoshida, K., \& Nishimura, J. 2004, ApJ, 601, 340

Large, M. I., Vaughan, A. E., \& Mills, B. Y. 1968, Nature, 220, 340

Manchester, R. N., Hobbs, G. B., Teoh, A., \& Hobbs, M. 2005, AJ, 129, 1993

Mangano, V., Massaro, E., Bocchino, F., Mineo, T., \& Cusumano, G. 2005, A\&A, 436, 917

Markwardt, C. B. \& Ögelman, H. 1995, Nature, 375, 40

Markwardt, C. B. \& Ögelman, H. B. 1997, ApJ, 480, L13

Moriguchi, Y., Yamaguchi, N., Onishi, T., Mizuno, A., \& Fukui, Y. 2001, PASJ, 53, 1025

Nishimura, J., Fujii, M., Taira, T., et al., 1980, ApJ, 238, 394

Profumo, S. 2008, ArXiv e-prints

Shen, C. S. 1970, ApJ, 162, L181+

van der Swaluw, E., Downes, T. P., \& Keegan, R. 2004, A\&A, 420, 937

van der Swaluw, E. \& Wu, Y. 2001, ApJ, 555, L49+

Weiler, K. W. \& Panagia, N. 1980, A\&A, 90, 269

Yüksel, H., Kistler, M. D., \& Stanev, T. 2009, Physical Review Letters, 103, 051101 


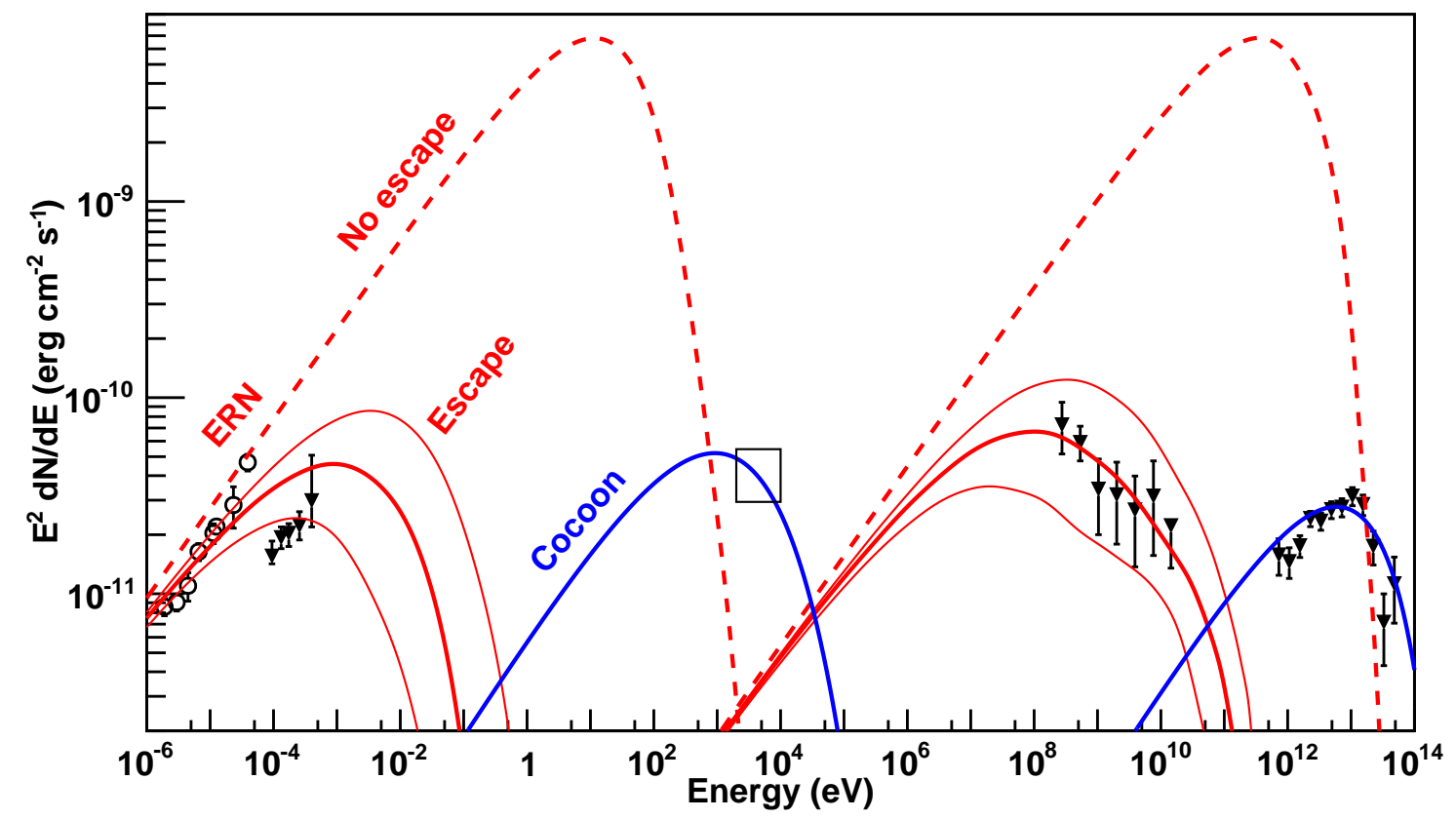

Fig. 2.- Measured SEDs for the cocoon/TeV nebula (blue) and the ERN (red) compared to model curves. Radio data are from Alvarez et al. (2001), WMAP, ASCA and Fermi-LAT from Abdo et al. (2010) and TeV from Aharonian et al. (2006). The total flux of the TeV nebula is $\sim 30 \%$ higher(Dubois et al. 2009), but the X-ray flux in this larger region is not well constrained. The magnetic field in the cocoon was chosen to be $4.5 \mu \mathrm{G}$. Injection from the hard X-ray nebula into this region follows a power-law with index -2 and cutoff at $70 \mathrm{TeV}$. The dashed line shows the ERN emission expected in the absence of escape and the solid curves for energy-dependent escape with $\delta=1000,2000$ (thick line) and 4000 times faster than Bohm diffusion. The current-day magnetic field is taken as $4 \mu \mathrm{G}$. The curves assume a birth-period of $P_{0}=22 \mathrm{~ms}$ and efficiency $\epsilon=0.3$ (almost identical results are obtained for $P_{0}=30 \mathrm{~ms}$ and $\left.\epsilon=0.5\right)$. 

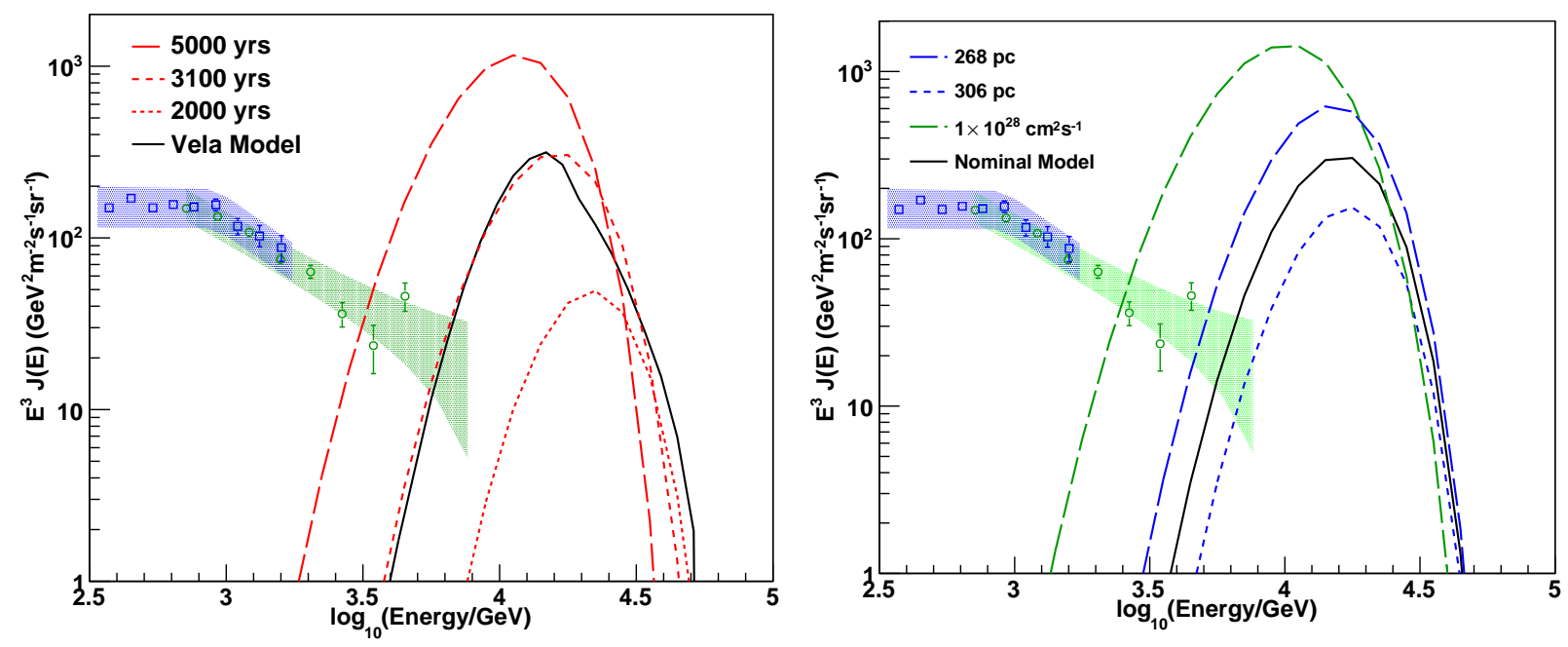

Fig. 3.- Left: Predicted electron spectrum from Vela X using the time dependent injection model (black). Simple burst-like power-law injection spectra are shown for comparison assuming different injection times (all $\mathrm{d}=290 \mathrm{pc}$, and a diffusion coefficient at $10 \mathrm{GeV}$ of $5 \times 10^{27} \mathrm{~cm}^{2} \mathrm{~s}^{-1}$ with energy dependence of $\mathrm{E}^{0.6}$ ). Right: Effects of varying distance (blue) or diffusion coefficient (green) for burst-like injection (at 3100 years). Data points show the H.E.S.S. electron measurements (Aharonian et al. 2008; Egberts et al. 2011). Shaded areas represent the systematic uncertainties. 